\title{
CREEP-VELOCITY BOUNDS AND GLACIER-FLOW PROBLEMS
}

\author{
By Andrew C. Palmer \\ (Department of Mechanical Engineering, University of Liverpool, Liverpool, England)
}

\begin{abstract}
A general result due to Martin can be used to find upper and lower bounds on velocities in steady-creep problems. This method can be applied to glacier flow if ice can be assumed to satisfy a powerlaw stress-strain-rate relation. Bounds on the mean velocity over the glacier cross-section and on the mean velocity on the surface are determined for a particular example (a uniform parabolic channel, with powerlaw exponent 3 ) and they are shown to bound quite closely the exact solutions due to Nye. Bounds can be found rapidly by hand calculation. The method can be applied to real glacier cross-sections measured in the field.
\end{abstract}

RÉsumé. Limites fluage-vitesse et problèmes de l'écoulement des glaciers. Un résultat général dû à Martin peut être utilisé pour trouver les limites supérieures et inférieures des vitesses dans les problèmes de fluage stationnaire. Cette méthode peut être appliquée à l'écoulement des glaciers si l'on peut admettre que la glace satisfait à une fonction de puissance entre la tension et la vitesse de déformation. Les limites de la vitesse moyenne d'un section transversale d'un glacier et de la vitesse moyenne à la surface sont déterminées pour un exemple particulier (lit uniforme parabolique, avec une fonction de puissance 3 ) et elles approchent de très près les solutions exactes de Nye. Les limites peuvent être obtenues rapidement par un calcul manuel. La méthode peut être appliquée aux sections transversales de glaciers réels mesurées in situ.

Zusammenfassung. Schranken der Kriechgeschwindigkeit und Probleme der Gletscherbewegung. Ein allgemeiner Ansatz von Martin kann zur Bestimmung der oberen und unteren Schranke der Geschwindigkeit bei Problemen des stetigen Kriechens benutzt werden. Diese Methode kann auf die Gletscherbewegung unter der Voraussetzung angewandt werden, dass die Deformationsgeschwindigkeit einem Potenzgesetz folgt. Die Schranken der mittleren Geschwindigkeit in einem Querschnitt des Gletschers und der mittleren Geschwindigkeit an der Oberfläche werden für ein spezielles Beispiel (gleichförmig parabolisches Gletscherbett, Exponent 3 für das Potenzgesetz) bestimmt und es wird gezeigt, dass sie sehr nahe an der exakten Lösung nach Nye liegen. Die Schranken können rasch durch Überschlagsrechnung gefunden werden. Die Methode ist zur Anwendung auf Gletscher-Querschnitte, die im Felde gemessen werden, geeignet.

\section{INTRODUCTION}

In a recent paper, Nye ( 1965$)$ has extended the existing theory of a valley glacier to take into account drag by the sides of the valley. He analysed the steady rectilinear flow of ice down a channel with uniform cross-section and uniform slope, and in his theoretical model assumed a power-law stress-strain-rate relation for steady creep consistent with Glen's (1955) experiments. The glacier was assumed not to slip over its bed, but Nye pointed out that this effect could be included in the theory when more was known about it, and that several of his results appear to hold independently of whether or not slip occurs. Analytical solutions exist only for four special channel cross-sections (channels infinitely wide with uniform depth, semi-circular, infinitely deep with uniform width, and slightly elliptical), although through a careful use of symmetry and dimensional arguments some useful results on flow in elliptical channels can be found.

Further progress requires numerical solution of the governing differential equations, using a digital computer, and in this way Nye found stress and velocity distributions for a number of distinct symmetrical channel shapes (rectangular, parabolic and semi-elliptical) of different proportions. Although this method gives very complete information, its use requires considerable programming effort and a large amount of computer time.

In the present paper we apply to glacier-flow problems a theorem due to Martin (ig66) for obtaining upper bounds on displacement rates in steady creep, and a development of Martin's approach to find lower bounds (Palmer, in press). Through this method one can find close upper and lower bounds on the mean velocity over the cross-section of the glacier, on the mean velocity on the surface and on certain other velocities. It can be applied to glaciers of arbitrary cross-section, not necessarily symmetrical, and to any power-law stressstrain-rate relation, whether the exponent is integral or non-integral. Close bounds can be found rapidly by comparatively simple hand calculations, or computed still more quickly 
using standard programmes. In no way, of course, can this method replace exact numerical solutions; it tells us nothing of the stress or of the fine details of the velocity distribution, and it gives only bounds on mean velocities, although these bounds are frequently close enough for most purposes. It is suggested here, however, that close bounds on mean velocities will yield useful information about real glaciers and that, because it makes trial solutions for different assumed flow laws much less laborious, this method may be helpful in linking field observations with the results of laboratory tests.

\section{Notation: General Theorems}

In order that the bound theorems can be stated concisely, Cartesian tensor notation will be used in the description of velocities, stresses and strain-rates. The position of a point is defined by its Cartesian coordinates $x_{1}, x_{2}, x_{3}$, and the velocity by its components $u_{i}(i=\mathrm{I}, 2,3)$ in the $\mathrm{I}, 2$ and 3 directions. The strain-rate $\dot{\epsilon}_{i j}(i, j=\mathrm{I}, 2,3)$ is defined by

$$
\dot{\epsilon}_{i j}=\frac{1}{2}\left(\frac{\partial u_{i}}{\partial x_{j}}+\frac{\partial u_{j}}{\partial x_{i}}\right) \text {. }
$$

Stress is described by $\sigma_{i j}(i, j=\mathrm{I}, 2,3)$, so that $\sigma_{1 \mathrm{I}}$ is the component of stress acting in the I direction on a plane normal to the 1 axis, $\sigma_{12}$ the component of stress acting in the 2 direction on a plane normal to the $\mathrm{I}$ axis, and so on. Body forces, referred to unit volume, are denoted $F_{i}$; surface tractions, referred to unit area, are denoted $T_{i}$. The repeated subscript summation convention (Prager, I96I) is used frequently, so that

$$
\begin{aligned}
T_{i} u_{i} & \equiv T_{1} u_{1}+T_{2} u_{2}+T_{3} u_{3}, \\
\sigma_{i j} \dot{\epsilon}_{i j} & \equiv \sigma_{11} \dot{\epsilon}_{11}+\sigma_{12} \dot{\epsilon}_{12}+\sigma_{13} \dot{\epsilon}_{13}+\sigma_{21} \dot{\epsilon}_{21}+\ldots+\sigma_{33} \dot{\epsilon}_{33}, \\
\sigma_{k k} & \equiv \sigma_{11}+\sigma_{22}+\sigma_{33} .
\end{aligned}
$$

Martin (1964, 1966) considered steady creep in materials whose stress-strain-rate law has the form

$$
\frac{\dot{\epsilon}_{i j}}{\dot{\epsilon}_{\mathrm{o}}}=\phi^{n} \frac{\partial \phi}{\partial\left(\sigma_{i j} / \sigma_{\mathrm{o}}\right)},
$$

where $\dot{\epsilon}_{\mathrm{o}}, \sigma_{\mathrm{o}}$ and $n$ are constants, $\phi\left(\sigma_{i j} / \sigma_{\mathrm{o}}\right)$ is homogeneous of degree one, and $\phi^{n+\mathrm{r}}$ is a convex function of its argument. As a special case of a more general result, he showed that for a body composed of such a material

$$
\frac{\mathrm{I}}{n+\mathrm{I}} \int_{V} \sigma_{i j}{ }^{s} \dot{\epsilon}_{i j}{ }^{s} d V+\frac{n}{n+\mathrm{I}} \int_{V} \sigma_{i j}{ }^{\sigma} \dot{\epsilon}_{i j}{ }^{o} d V \geqslant \int_{\boldsymbol{A}} T_{i}^{s} u_{i}{ }^{o} d A+\int_{V} F_{i}{ }^{s} u_{i}{ }^{c} d V,
$$

where $\sigma_{i j}{ }^{s}, T_{i}^{s}$ and $F_{i}^{s}$ are any set of stresses, surface tractions and body forces which satisfy equilibrium, and $u_{i}{ }^{c}, \dot{\epsilon}_{i j}{ }^{c}$ any compatible set of velocities and strain-rates. The integrals are taken over the volume $V$ and surface area $A$ of the body. Here $\dot{\epsilon}_{i j}{ }^{s}$ is related to $\sigma_{i j}{ }^{s}$ only through the stress-strain-rate relation (3), and need not be integrable; $\sigma_{i j}{ }^{c}$ is related to $\dot{\epsilon}_{i j}{ }^{C}$ only through (3), and need not satisfy equilibrium. In addition, from virtual work

$$
\int_{V} \sigma_{i j}{ }^{*} \dot{\epsilon}_{i j}^{* *} d V=\int_{\boldsymbol{A}} T_{i}^{*} u_{i}^{* *} d A+\int_{V} F_{i}^{*} u_{i}^{* *} d V
$$

for any $\sigma_{i j}{ }^{*}, T_{i}^{*}, F_{i}^{*}$ which are in equilibrium, and any compatible $u_{i}^{* *}, \dot{\epsilon}_{i j}^{* *}$. This inequality can be used to find bounds on velocities in the solution of the following mixed boundary-value problem: surface tractions $T_{i}$ are prescribed on one part $A_{T}$ of the boundary, while on the remainder $A_{V}$ the velocity $u_{i}$ is zero; body forces $F_{i}$ are prescribed throughout the body. In what follows quantities $u_{i}, \dot{\epsilon}_{i j}, \sigma_{i j}, T_{i}, F_{i}$ without any superscript will refer to the solution of this problem. 
Since the solution velocities and strain-rates certainly satisfy the conditions imposed on $u_{i}^{c}, \dot{\epsilon}_{i j}{ }^{c}$ we can set

$$
\begin{aligned}
u_{i}{ }^{c} & =u_{i}, \\
\dot{\epsilon}_{i j}{ }^{c} & =\dot{\epsilon}_{i j}
\end{aligned}
$$

throughout (4). Applying (5) to the solution velocity and stress fields, substituting into (4) and re-arranging (Martin, I966)

$$
\frac{\mathrm{I}}{n+\mathrm{I}} \int_{V} \sigma_{i j}{ }^{s} \dot{\epsilon}_{i j}{ }^{s} d V \geqslant \int_{A}\left(T_{i}^{s}-\frac{n}{n+\mathrm{I}} T_{i}\right) u_{i} d A+\int_{V}\left(F_{i}^{s}-\frac{n}{n+\mathrm{I}} F_{i}\right) u_{i} d V .
$$

This inequality gives upper bounds on velocities. If instead we identify $\sigma_{i j}{ }^{s}, T_{i}^{s}$ and $F_{i}{ }^{s}$ with corresponding quantities in the solution of the boundary-value problem, which must certainly satisfy equilibrium, if, that is

$$
\begin{aligned}
\sigma_{i j}{ }^{s} & =\sigma_{i j}, \\
T_{i}{ }^{s} & =T_{i}
\end{aligned} \quad F_{i}^{s}=F_{i}, \quad \dot{\epsilon}_{i j}{ }^{s}=\dot{\epsilon}_{i j} \quad \text { in } V,
$$

then we can again apply (5) to the solution velocity and stress fields, substitute in (4) and re-arrange, to arrive at

$$
\frac{n}{n+\mathrm{I}} \int_{V} \sigma_{i j}{ }^{c} \dot{\epsilon}_{i j}{ }^{c} d V \geqslant \int_{\boldsymbol{A}} T_{i}\left(u_{i}^{c}-\frac{\mathrm{I}}{n+\mathrm{I}} u_{i}\right) d A+\int_{\boldsymbol{V}} F_{i}\left(u_{i}{ }^{\sigma}-\frac{\mathrm{I}}{n+\mathrm{I}} u_{i}\right) d V .
$$

In the glacier-flow problem about to be considered $\int_{A} T_{i}\left(u_{i}^{c}-\frac{\mathrm{I}}{n+\mathrm{I}} u_{i}\right) d A$ is zero for a wide class of admissible velocity fields $u_{i}{ }^{c}$; it follows that (9) can be used directly to find a lower bound on the mean velocity over the glacier cross-section.

\section{Theoretical Model}

Ice flows steadily under gravity down a straight uniform channel of uniform slope $\alpha$. Position is defined by coordinate axes aligned in the manner illustrated in Figure $\mathrm{I}$; the I-axis is directed along the channel, at an angle $\alpha$ to the horizontal. If the channel is symmetrical the $x_{1}$ and $x_{2}$ axes lie in the plane of symmetry; otherwise their position is chosen arbitrarily. The plane $x_{2}=0$ coincides with the upper surface of the ice.

Stated in terms of a simple shear deformation in which each material point has a velocity only in the I-direction, Glen's stress-strain-rate relation takes the form

$$
\frac{\partial u_{1}}{\partial x_{2}}=C \tau^{n-1} \sigma_{12}, \quad \frac{\partial u_{1}}{\partial x_{3}}=C \tau^{n-1} \sigma_{13},
$$
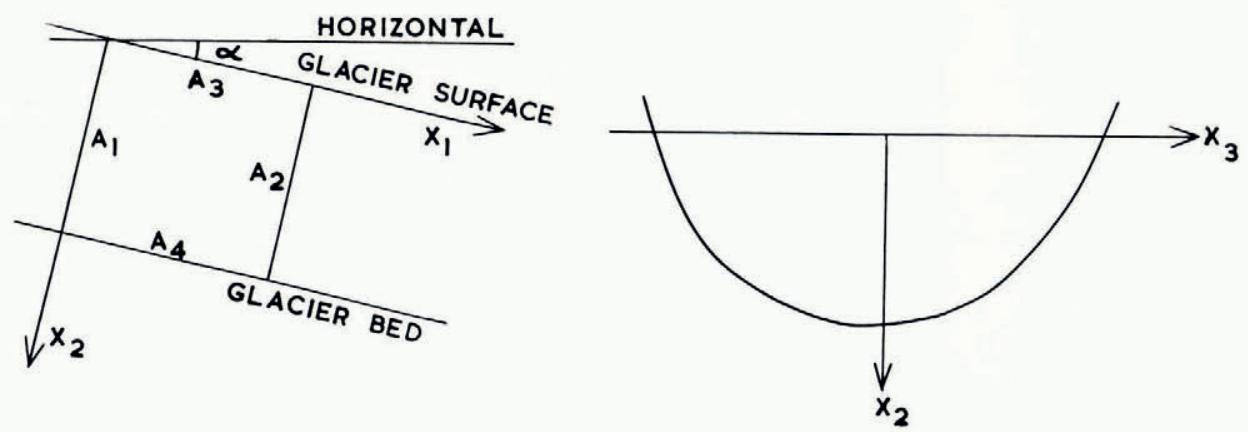

Fig. $I$ 
where $C$ and $n$ are constants and $\tau=\left(\sigma_{12}{ }^{2}+\sigma_{13}\right)^{\frac{1}{2}}$. A generalization of this which relates the stress to the strain-rate for an arbitrary deformation is

$$
\dot{\epsilon}_{i j}=\frac{1}{2} C\left(\frac{1}{2} s_{i j} s_{i j}\right)^{(n-\mathrm{r}) / 2} s_{i j} \text {, }
$$

where $s_{i j}$ is the deviatoric stress tensor $\sigma_{i j}-\frac{1}{3} \sigma_{k k} \delta_{i j}$; in this $\delta_{i j}$ is the Kronecker delta, $\delta_{i j}=\mathrm{I}$ when $i=j, \delta_{i j}=0$ when $i \neq j$. This is an appropriate form for an incompressible material. Note, however, that in what follows the stress-strain-rate relation is applied only to stress fields which give velocity distributions of the "simple shear" pattern to which (2) refers. As Nye has pointed out, it is not necessary in this problem to assume a full generalization of the flow law for simple shear.

\section{UPPER BOUNDS}

The upper and lower bound inequalities are now applied in turn to a section of the glacier cut between two planes unit distance apart and parallel to the $x_{2}, x_{3}$ plane (Fig. I). This section is bounded by the following surfaces: the up-stream cross-section of the glacier (denoted $A_{\mathrm{I}}$ ), the down-stream cross-section (denoted $A_{2}$ ), the part of the glacier surface lying between the two sectioning planes (denoted $A_{3}$ ), and the corresponding part of the glacier bed (denoted $A_{4}$ ). The ice is acted on by a gravity force $\rho g$ on unit volume, where $\rho$ is the density and $g$ the acceleration due to gravity; resolving this in the coordinate directions, the body-force components $F_{i}$ are

$$
\begin{aligned}
& F_{1}=\rho g \sin \alpha, \\
& F_{2}=\rho g \cos \alpha, \\
& F_{3}=0 .
\end{aligned}
$$

If a stress field satisfies equilibrium, at each point

$$
\frac{\partial \sigma_{i j}}{\partial x_{j}}+F_{i}=0
$$

Since the upper surface of the glacier is free,

$$
\tau_{i}=\text { o on } A_{3} \text {. }
$$

From the assumed condition that there is no slip between the glacier and its bed

$$
u_{i}=0 \text { on } A_{4} \text {. }
$$

Since each section of the glacier is identical, in the sense that the flow is uniform along its length, the surface traction at a point on the up-stream face $A_{\mathrm{I}}$ is equal and opposite to that at the corresponding point on the down-stream face $A_{2}$, and the velocities at the two points are identical. It follows that

$$
\int_{A_{\mathrm{x}}} T_{i} u_{i} d A+\int_{A_{2}} T_{i} u_{i} d A=0 .
$$

In order to use the inequalities, we have to choose the stress field $\sigma_{i j}{ }^{S}$-which only has to satisfy equilibrium - in such a way that "unwanted" velocity components do not appear; this can be done by choosing $T_{i}^{S}$ and $F_{i}^{S}$ so that the multipliers of unwanted velocities in (7) vanish. If we set $F_{i}^{s}=F_{i}$, choose any equilibrium stress field which satisfies

$$
\begin{aligned}
T_{i}^{s}=0 & \text { on } A_{3}, \\
\frac{\partial \sigma_{i j}{ }^{s}}{\partial x_{j}}+F_{i}{ }^{s}=0 & \text { in } V,
\end{aligned}
$$

and take account of the boundary conditions expressed in (I4), (I5) and (I6), it follows from (7) that

$$
\int \sigma_{i j}{ }^{s} \dot{\epsilon}_{i j}{ }^{s} d V \geqslant \int F_{i} u_{i} d V
$$


But since the section we are considering has unit length in the flow direction

$$
\int F_{i} u_{i} d V=\int(\rho g \sin \alpha) u_{\mathrm{I}} d V=(\rho g \sin \alpha) \bar{u} A^{*},
$$

where $A^{*}$ is the cross-sectional area of the glacier and $\bar{u}$ is the mean velocity over the crosssection. ${ }^{\dagger}$ Thus

$$
\bar{u} \leqslant \frac{\mathrm{I}}{(\rho g \sin \alpha) A^{*}} \int_{V} \sigma_{i j}{ }^{s} \dot{\epsilon}_{i j}{ }^{s} d V
$$

If we let

$$
\begin{aligned}
& \sigma_{11}{ }^{s}=\sigma_{22}{ }^{s}=\sigma_{33}{ }^{s}=-\rho g x_{2} \cos \alpha, \\
& \sigma_{12}{ }^{s}=-\rho g \beta x_{2} \sin \alpha, \\
& \sigma_{13}{ }^{s}=-\rho g(\mathrm{I}-\beta) x_{3} \cos \alpha,
\end{aligned}
$$

where $\beta$ is a constant still to be found, the equilibrium and boundary conditions on $\sigma_{i j}{ }^{s}$ are satisfied. These stresses produce a simple shear deformation

$$
\begin{aligned}
& \dot{\epsilon}_{\mathrm{I} 3}=-\frac{1}{2}(\rho g \sin \alpha)^{n}\left\{(\mathrm{I}-\beta)^{2} x_{3}{ }^{2}+\beta^{2} x_{2}{ }^{2}\right\}^{(n-\mathrm{I}) / 2}(\mathrm{I}-\beta) C x_{3}, \\
& \dot{\epsilon}_{\mathrm{I} 2}=-\frac{1}{2}(\rho g \sin \alpha)^{n}\left\{(\mathrm{I}-\beta)^{2} x_{3^{2}}+\beta^{2} x_{2}\right\}^{(n-\mathrm{I}) / 2} \beta C x_{2} .
\end{aligned}
$$

Then, from inequality $(20)$,

$$
\bar{u} \leqslant \frac{C}{A^{*}}(\rho g \sin \alpha)^{n} \int_{A_{I}}\left\{(\mathrm{I}-\beta)^{2} x_{3}^{2}+\beta^{2} x_{2}^{2}\right\}^{(n+1) / 2} d x_{2} d x_{3} ;
$$

an upper bound on the mean velocity in a channel of arbitrary cross-section. In order to compare it with Nye's exact numerical solution, we apply it to a symmetrical parabolic channel of depth $a$ and half-width $W a$, and let $n=3$. Evaluating the integral,

$$
\bar{u} \leqslant \frac{3}{35} a^{4} C(\rho g \sin \alpha) 3\left\{(\mathrm{I}-\beta)^{4} W^{4}+\frac{16}{2} \frac{6}{7} \beta^{2}(\mathrm{I}-\beta)^{2} W^{2}+\frac{128}{99} \beta^{4}\right\} .
$$

If $\beta=\mathrm{I}$, we have an upper bound

$$
\bar{u} \leqslant \frac{128}{1155} a^{4} C(\rho g \sin \alpha) 3
$$

identical to the solution for an infinitely wide parabolic channel (Nye, 1965). If $\beta=0$,

$$
\bar{u} \leqslant \frac{3}{35} a^{4} C(\rho g \sin \alpha) 3 W^{4}
$$

which approaches the exact solution as $W \rightarrow$ o. Although any value of $\beta$ gives an upper bound on $\bar{u}$, one naturally seeks to minimize this bound through an appropriate choice of $\beta$; this is found by differentiating (24) with respect to $\beta$, setting the derivative zero, and for each $W$ of interest solving the resulting cubic equation in $\beta /(\mathrm{I}-\beta)$. In Table I and Figure 2 the bounds found by this procedure are compared with values from the numerical solutions.

Although more complex stress fields than (2 I) can be applied in a search for even closer bounds, further improvement is only gained after much more extensive calculation, which is hardly repaying. It is interesting that such good bounds are given by this simple stress field, in which the shear stress on the surface increases steadily towards the edge. The exact solution of the problem shows that the actual stress distribution is quite different, and that the shear stress at the surface reaches a maximum at about $0.6 \mathrm{Wa}$ from the centre line and thereafter decreases.

$\dagger$ Since the $F_{i}$ are the same at each point, this would hold true even if the velocity components $u_{2}$ and $u_{3}$ perpendicular to the channel direction did not vanish. If the flow pattern is uniform along the length of the glacier, there can be no net flow in the 2- and 3 -directions, and so $\int F_{2} u_{2} d V$ and $\int F_{3} u_{3} d V$ vanish. 
Table I. Bounds on Dimensionless Mean Velocity $\bar{u} / a 4 C(\rho g \sin \alpha) 3$ for Parabolic Channels $(n=3)$ with Different Values of $W$, the Half-wid'th to Depth Ratio

\begin{tabular}{|c|c|c|c|}
\hline$W$ & Lower bound & $\begin{array}{c}\bar{u} \mid a 4 C(\rho g \sin \alpha) 3 \\
\text { Exact } \\
(\text { Nye, I } 965)\end{array}$ & Upper bor \\
\hline I & 0.0062 & o.0 149 & o.01 53 \\
\hline 2 & 0.0286 & $0.044^{\circ}$ & $0 \cdot 0463$ \\
\hline 3 & 0.0497 & 0.0637 & o. 0673 \\
\hline 4 & 0.0648 & 0.0757 & 0.0801 \\
\hline$\infty$ & $0 \cdot 1108$ & $0 \cdot 1108$ & o. I I 08 \\
\hline
\end{tabular}

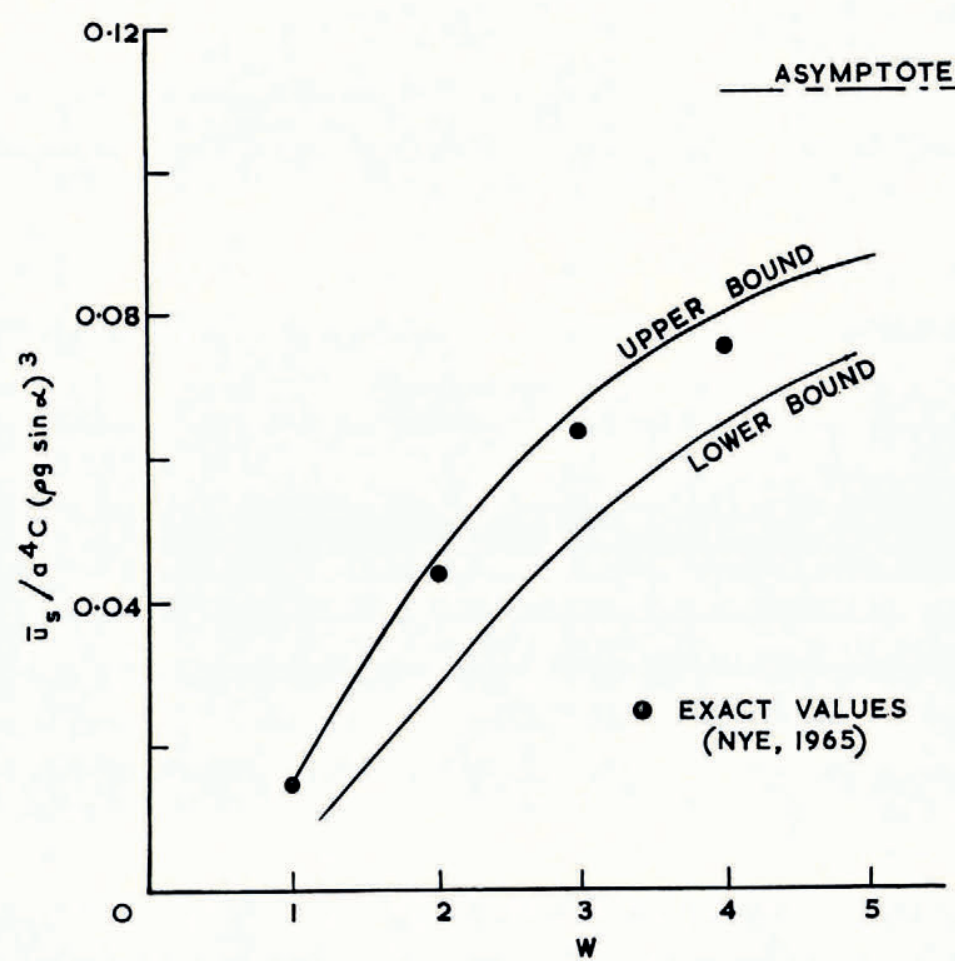

Fig. 2. Upper and lower bounds on $\bar{u}$, the mean velocity, as functions of $W$, the ratio of half-width to depth, for parabolic channels

In order to find an upper bound on the mean velocity on the upper surface of the glacier, a different choice of $\sigma_{i j}{ }^{s}$ in inequality (7) is required. If we let

$$
\begin{array}{lrlrl}
T_{\mathrm{I}}{ }^{s} & =\gamma \psi a\left(\frac{n}{n+\mathrm{I}} \rho g \sin \alpha\right), & {T_{2}}^{s}=T_{3}^{s}=o & \text { on } A_{3}, \\
F_{i}^{s}=\frac{n}{n+\mathrm{I}} F_{i} & \text { in } V,
\end{array}
$$

where $\gamma$ and $\psi$ are positive constants still to be determined, then, if we take account of (I4), (15) and (I6), inequality (7) reduces to

$$
\frac{\mathrm{I}}{n+\mathrm{I}} \int_{V} \sigma_{i j}{ }^{s} \dot{\epsilon}_{i j}{ }^{s} d V \geqslant \int_{A_{3}} \gamma \psi a\left(\frac{n}{n+\mathrm{I}} \rho g \sin \alpha\right) u_{\mathrm{I}} d A=2 W \gamma \psi a^{2}\left(\frac{n}{n+\mathrm{I}} \rho g \sin \alpha\right) u_{\mathrm{s}} \text {. }
$$


Here $u_{\mathrm{s}}$ is the mean velocity on the surface, $a$ is the depth of the channel on the centre line (or at some arbitrarily chosen point) and $W$ the half-width/depth ratio. Then

$$
u_{s} \leqslant \frac{\mathrm{I}}{2 n W a^{2} \gamma \psi} \int_{V} \sigma_{i j}{ }^{s} \dot{\epsilon}_{i j}{ }^{s} d V .
$$

A simple $\sigma_{i j}{ }^{s}$ stress field which satisfies (27) and the equilibrium condition (13) is

$$
\begin{aligned}
& {\sigma_{12}}^{s}=-\gamma\left(\frac{n}{n+\mathrm{I}} \rho g \sin \alpha\right)\left(x_{2}+\psi a\right), \\
& {\sigma_{13}}^{s}=-(\mathrm{I}-\gamma)\left(\frac{n}{n+\mathrm{I}} \rho g \sin \alpha\right) x_{3}, \\
& {\sigma_{1 \mathrm{I}}}^{s}={\sigma_{22}}^{s}={\sigma_{33}}^{s}=-\frac{n}{n+\mathrm{I}} \rho g x_{2} \cos \alpha, \\
& {\sigma_{23}}^{s}=0 .
\end{aligned}
$$

This stress distribution again corresponds to a simple shear deformation; the strainrates are given by (10). Applying this to the parabolic cross-section described earlier, and again letting $n=3$,

$$
\begin{gathered}
u_{8} \leqslant \frac{1}{4} a^{4} C(\rho g \sin \alpha) 3\left\{\frac{\gamma^{3}}{\psi}\left(\frac{256}{3465}+\frac{128}{315} \psi+\frac{32}{35} \psi^{2}+\frac{16}{15} \psi^{3}+\frac{2}{3} \psi^{4}\right)+\right. \\
\left.+\frac{4}{15} \frac{\gamma(\mathrm{I}-\gamma)^{2}}{\psi} W^{2}\left(\frac{8}{63}+\frac{4}{7} \psi+\psi^{2}\right)+\frac{2}{35} \frac{(\mathrm{I}-\gamma)^{4}}{\gamma \psi} W^{4}\right\} .
\end{gathered}
$$

This bound can now be optimized for each $W$ by an appropriate choice of $\gamma$ and $\psi$; the smallest upper bound can be found either analytically or by one of the standard methods for optimum search (Wilde, I964). A close bound can be located quite rapidly, since near to the optimum the expression (3I) alters only slowly with changes in $\gamma$ and $\psi$. A comparison between upper bounds on $u_{\mathrm{s}}$ and the exact solution is given in Table II and Figure 3 .

This technique can also be used to find a direct bound on $u_{8}-\bar{u}$, or on the mean velocity of any region (such as the middle third of the glacier surface), but not to bound the velocity of single points. This would require a dummy concentrated force at the point, which would give rise to a singularity in the dissipation function.

\section{LOWER Bounds}

A lower bound on the mean velocity $\bar{u}$ can be derived from inequality (9). If the velocity field $u_{i}^{c}$ satisfies the condition that there is no slip on the glacier bed, and is independent of $x_{\mathrm{I}}$, it follows that

and

$$
\begin{aligned}
\int_{A_{x}} T_{i} u_{i}^{c} d A+\int_{A_{2}} T_{i} u_{i}^{c} d A & =0, \\
u_{i}^{c} & =0 \quad \text { on } A_{4},
\end{aligned}
$$

Inequality (9) then gives

$$
\int_{A} T_{i}\left(u_{i}^{c}-\frac{\mathrm{I}}{n+\mathrm{I}} u_{i}\right) d A=\mathrm{o} .
$$

$$
\begin{gathered}
\int_{V} F_{i} u_{i} d V \geqslant(n+\mathrm{I}) \int_{V} F_{i} u_{i}^{c} d V-n \int \sigma_{i j}{ }^{c} \dot{\epsilon}_{i j}{ }^{c} d V, \\
(\rho g \sin \alpha) \bar{u} A^{*} \geqslant(n+\mathrm{I}) \int_{V}(\rho g \sin \alpha) u_{\mathrm{I}}^{c} d V-n \int \sigma_{i j}{ }^{c} \dot{\epsilon}_{i j}{ }^{c} d V .
\end{gathered}
$$


Table II. Upper Bound on Dimensionless Surface Velogity $u_{\mathrm{S}} / a 4 C(\rho g \sin \alpha) 3$ for Parabolic Channels $(n=3)$ With Different Values of $W$

\begin{tabular}{|c|c|c|}
\hline \multirow[b]{2}{*}{$W$} & \multicolumn{2}{|c|}{$u_{\mathrm{S}} / a 4 C\left(\rho g \sin \alpha^{\prime}\right)^{3}$} \\
\hline & $\begin{array}{c}\text { Exact } \\
(\text { Nye, I965) }\end{array}$ & Upper bound \\
\hline $\mathbf{I}$ & 0.0178 & 0.0180 \\
\hline 2 & 0.0449 & $0.049 \mathrm{I}$ \\
\hline 3 & 0.0639 & 0.0679 \\
\hline 4 & 0.0753 & 0.0802 \\
\hline$\infty$ & 0.1016 & $0 \cdot 1057$ \\
\hline
\end{tabular}

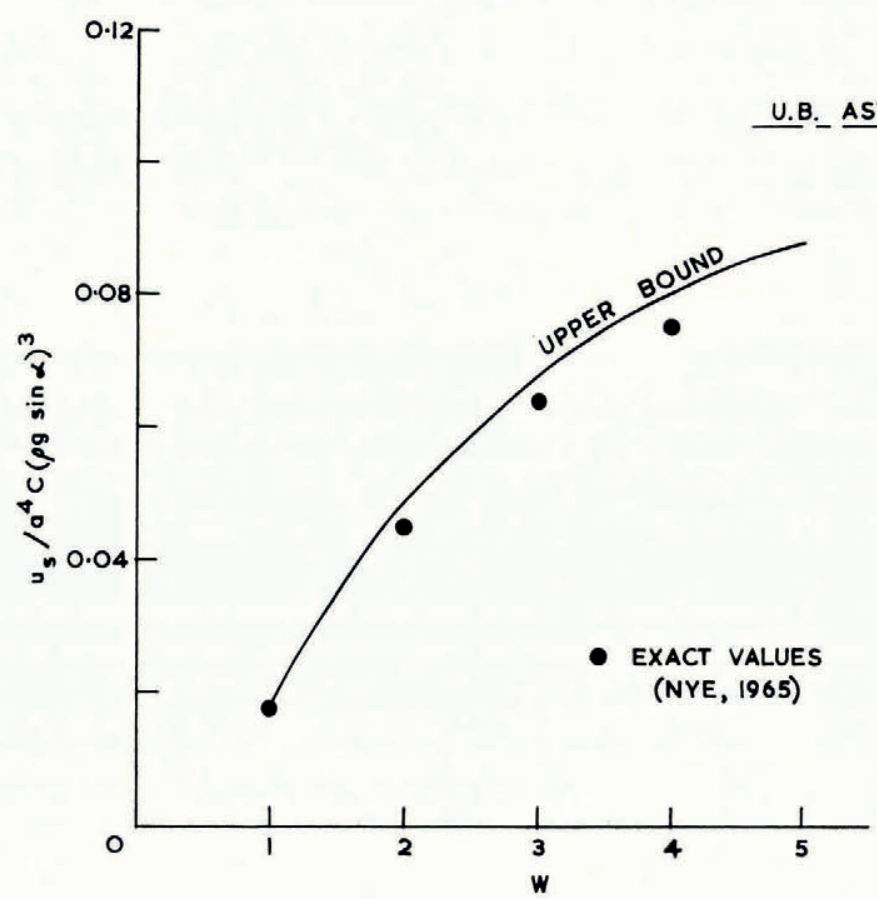

Fig. 3. An upper bound on $u_{\mathrm{s}}$, the mean velocity on the surface, as a function of $W$, the ratio of half-width to depth, for parabolic channels

Any velocity field which satisfies (34) then gives a lower bound on $\bar{u}$; the strain-rate $\dot{\epsilon}_{i j}$ is derived from (I), and by inverting the stress-strain-rate relation it can be shown that

$$
\sigma_{i j} \dot{\epsilon}_{i j}=C^{-1 / n}\left(2 \dot{\epsilon}_{i j} \dot{\epsilon}_{i j}\right)^{(n+\mathrm{r}) / 2 n} \text {. }
$$

We again apply this method to find a lower bound on $\bar{u}$ in a parabolic channel when $n=3$. If the half-width/depth ratio were large, the surface velocity at a point where the depth is $H$ would be the same as that in an infinitely wide channel of uniform depth $H$, and since $n=3$ this velocity is proportional to $H^{4}$. The boundary of the channel is the parabola

$$
\frac{x_{2}}{a}=\mathrm{I}-\left(\frac{x_{3}}{W a}\right)^{2} \text {. }
$$

If the velocity on the centre line at the surface is $\lambda$ and a velocity distribution identical to that in a very wide channel is chosen for $u_{i}{ }$,

$$
\begin{aligned}
& u_{\mathrm{I}}{ }^{c}=\lambda\left\{\left(\mathrm{I}-\left[\frac{x_{3}}{W a}\right]^{2}\right)^{4}-\left(\frac{x_{2}}{a}\right)^{4}\right\} \\
& u_{2}{ }^{c}=u_{3}{ }^{c}=0 .
\end{aligned}
$$


Then

$$
\sigma_{i j} \dot{\epsilon}_{i j}{ }^{c}=C^{-1 / 3}\left(\frac{\lambda}{a}\right)^{4 / 3} \mathrm{I} 6^{2 / 3}\left\{\left(\frac{x_{2}}{a}\right)^{6}+\frac{4}{W^{2}}\left(\frac{x_{3}}{W a}\right)^{2}\left(1-\frac{x_{3}^{2}}{W^{2} a^{2}}\right)^{6}\right\}^{2 / 3}
$$

and from (35)

where

$$
\begin{gathered}
\frac{4}{3} W a^{2}(\rho g \sin \alpha) \bar{u} \geqslant \frac{8192}{3465} \lambda W a^{2}-6 C^{-1 / 3} \mathrm{I} 6^{2 / 3}\left(\frac{\lambda}{a}\right)^{4 / 3} W a^{6} I(W) \\
I(W)=\int_{0}^{1} \int_{0}^{1}\left(u^{6}+\frac{4 v^{2}}{W^{2}}\right)^{2 / 3}\left(\mathrm{I}-v^{2}\right)^{5} d u d v .
\end{gathered}
$$

Minimizing the right-hand side of (40) with respect to $\lambda$, and simplifying

$$
\bar{u} \geqslant \frac{128}{1155}\left(\frac{256}{3465 I(W)}\right)^{3}{ }^{4} C(\rho g \sin \alpha) 3 .
$$

If $W \rightarrow \infty, I(W) \rightarrow 256 / 3465$, and we have the exact solution, identical with the lower bound. Numerical integration of $I(W)$ for finite $W$ gives a lower bound on $\bar{u}$. This is compared with Nye's exact numerical solution and with the upper bound in Table I and Figure 2.

The lower bound on $\bar{u}$ represented by the right-hand side of inequality (40) is rather ill-conditioned, in the sense that it is a small difference between large quantities. As one might expect from this, these lower bounds are more sensitive to the choice of the velocity field $u_{i}^{c}$ than are the upper bounds found earlier to the choice of $\sigma_{i j}{ }^{s}$. Since even for $W=4$ the exact velocity distribution across the glacier width is rather different from that across an infinitely wide parabolic channel, the lower bound given by the velocity distribution $(38)$ is not very close. It is, however, close enough to give useful information about the effect of the valley sides.

Inequality (9) cannot be used directly to locate a lower bound on the mean surface velocity $u_{\mathrm{s}}$, since it is not possible to choose $u_{i}^{o}$ so that the unwanted velocity components do not appear. It is however possible to proceed in the following way: multiply (7) by a positive quantity $\Lambda$ (still to be determined), add to it inequality (9) and then re-arrange. The resulting inequality (Palmer, in press) contains both an unknown equilibrium stress field and an unknown velocity field. An appropriate choice of $T_{i}^{s}$ and $F_{i}^{s}$ then eliminates unwanted velocity components. If the velocity field is defined by one parameter $\gamma$, and the stress field by one parameter $\beta$, this gives a lower bound velocity which has to be minimized with respect to the three variables $\Lambda, \gamma$ and $\beta$. In the present problem the advantage in simplicity of the method is then lost, and accordingly it will not be pursued further.

\section{Application to Real Glaciers}

The technique for finding velocity bounds described in this paper can be applied to actual glaciers whose cross-sections have been determined by sounding. Finding upper bounds requires statically admissible stress fields which satisfy a boundary condition on the upper surface of the ice, whereas to find lower bounds one needs a kinematically admissible velocity field which satisfies the "no slip" boundary condition on the lower surface. Since the upper surface - or a reasonable idealization of it-will generally be the geometrically simpler, it will probably be easier to find upper bounds. If, for example, the upper surface is level in the cross-stream direction, the stress field (2I) is suitable. When the section is asymmetric, a coordinate origin can be chosen arbitrarily. If the stress-strain-rate exponent $n$ is 3 , a determination of a lower bound on $\bar{u}$ then requires only the integration of $x_{2}{ }^{4}, x_{3}{ }^{4}$ and $x_{2}{ }^{2} x_{3}{ }^{2}$ over the measured cross-section. The calculation is only a little more complicated for other values of $n$.

MS. received 9 August 1966 


\section{REFERENCES}

Glen, J. W. 1955. The creep of polycrystalline ice. Proceedings of the Royal Society, Ser. A, Vol. 228, No. I175, p. $519-38$.

Martin, J. B. 1964. A displacement bound technique for elastic continua subjected to a certain class of dynamic loading. Journal of the Mechanics and Physics of Solids, Vol. 12, No. 3, p. 165-75.

Martin, J. B. 1966. A note on the determination of an upper bound on displacement rates for steady creep problems. Fournal of Applied Mechanics, Vol. 33, No. 1, p. 216-17.

Nye, J. F. 1965. The flow of a glacier in a channel of rectangular, elliptic or parabolic cross-section. Journal of Glaciology, Vol. 5, No. 4I, p. 66I-90.

Palmer, A. C. In press. A lower bound on displacement rates in steady creep. Fournal of Applied Mechanics.

Prager, W. 1961. Introduction to mechanics of continua. Boston, Mass., Ginn.

Wilde, D. J. 1964. Optimum seeking methods. Englewood, N. J., Prentice-Hall. 